\title{
Key Requirements for Integrating Usability Engineering and Software Engineering
}

\author{
Karsten Nebe ${ }^{1}$ and Volker Paelke ${ }^{2}$ \\ ${ }^{1}$ University of Paderborn, C-LAB, Fuerstenallee 11, 33102 Paderborn, Germany \\ Karsten.Nebe@ $\mathrm{C}-1 \mathrm{ab}$.de \\ ${ }^{2}$ Institut de Geomàtica, Av. Carl Friedrich Gauss, 11, 08860 Castelldefels, Spain \\ Volker.Paelke@ideg.es
}

\begin{abstract}
To improve the integration between Software Engineering (SE) and Usability Engineering (UE) this paper identifies areas of overlap and develops proposals for their integration. The focus is on key requirements that were derived using semi-structured interviews and questionnaires. The principles and activities in the standards ISO 13407 and ISO/PAS 18152 were concretized to establish specific quality aspects. The identified requirements provide a foundation for systematic modification of existing development processes with established best practices from both disciplines.
\end{abstract}

Keywords: Integration, Assessment, Standards DIN EN ISO 13407 and ISO/PAS 18152, Process Models, Process Definition.

\section{Introduction}

Software development processes aim to produce products with a guaranteed quality level, where quality can refer to a variety of aspects including reliability and maintainability from a technical perspective as well as usability and adequateness from a usage perspective. Software Engineering (SE) has developed SE models as systematic approaches to address technical quality goals during development. SE models define detailed activities, the sequence in which these activities have to be performed, and the resulting deliverables and are meant to unify working processes [13]. To address the quality goals from the usage perspective the domain of Usability Engineering (UE) has also developed a variety of methods and processes. The development of high quality software requires to combine the goals and methods of SE and UE in a way that allows systematic implementation while considering all quality factors as well as costs and time. A key question is how this integration can be realized in practice. The introduction of novel development processes that address all quality requirements and integrate methods from both disciplines is problematic. In most development contexts a modification of existing SE processes is desired. The challenge is to provide systematic support for the assessment of existing SE processes and guidelines for their extension with usability oriented activities.

In the study described here the first aim was to identify an appropriate level of abstraction at which such guidance can be provided that is neither restricted to a specific process but concrete enough to add value to the integration process. The second goal 
was to provide concrete guidance through an assessment process at this level of abstraction.

Therefore the authors first analyzed various types of different integration approaches in order to identify the most promising level of abstraction for further integration strategies. The level of software engineering and usability standards is selected for closer inspections, i.e. in terms of assessment approaches. Previous work is summarized and the proceedings of an expert evaluation (interviews) are presented. The resulting requirements can be used first, as a basis for the definition of software development processes aiming at usable solutions; and second, as an enhancement to existing assessment standards. Then, first steps towards validation and application of these requirements are discussed.

\section{Integration Approaches}

In theory and praxis, a considerable number of integration approaches exist [15] (detailed analysis can be found at [10]). Some of these approaches tend to define common activities and artifacts for both SE and UE and to integrate these specific activities into the process of development. They aim at a 'soft integration' of UE aspects on a mutual basis, e.g. at interlinking relative results. Most approaches focus on a minimal organizational and structural transformation and/or change. For example, Schaffer [14] presents a method for the integration of UE activities, which is based on the evaluation of a concrete existing process.

Other integration approaches address the level of process definitions and process models. These aim to define pre-settings for the development and contain both a more concrete approach (focusing on the integration of UE activities in an already existing SE Models), and more fundamental aspects of process models (independently of any concrete SE Model). An example for approaches that integrate UE activities with existing SE Models is Pelka [12] who build on the V-Model as a base for the integration of UE activities.

A third group of integration approaches focuses at an even higher level of abstraction and describe organizational measures, principles, paradigms or metamodels. Those approaches are independent from any specific process model or activities but rather describe organizational measures, principles, paradigms or metamodels. As an example, Pawar [11] analyzed several activities of SE and UE in order to identify principal similarities as the basis for a framework for integration.

These approaches can be structured into three levels of abstraction [6]: The abstract level of standards, the level of process models and the operational process level. These are related in a hierarchy: standards define the overarching framework, process models describe systematic and traceable approaches within such a framework, and at the operational level the models are tailored to fit the specifics of an organization [6].

\section{Process Assessments}

This hierarchy exists in both disciplines software engineering and usability engineering and can be exploited for integration. At the level of standards general 
integration strategies can be defined that are applicable to a large number of existing development processes. For the purpose of integration we have focused on the SE standard ISO/IEC 12207 [2] and the UE standard ISO 13407 [1] . ISO/IEC 12207 defines a process framework for the development and management of software systems and identifies required processes and activities for the development-lifecycle. ISO 13407 defines four activities of human-centered design that should take place during system development.

An analysis [6, 7] shows that while some guidance can be derived from matching these standards, in practice more detailed and adequate criteria for the assessment are necessary to make objective statements about the ability of process models to create usable software and to derive actions for process improvement. We have therefore followed a process assessment approach that aims at the evaluation and optimization of existing processes in order to ensure technical quality and usability. Separate assessment approaches exist in SE and UE. ISO/IEC 15504 [3] describes a methodology and structure for assessing software development processes and defines requirements for the assessment, an assessment model and a reference model. For the domain of UE a similar approach exists with ISO/PAS 18152 [4]. To ensure usability within the development process there is a need for specific quality criteria for the assessment that integrates usability criteria.

The authors believe that there is such thing as a 'common understanding' in terms of what experts think of when they talk about UE and this is certainly represented by the definition of the human-centred-design process in the DIN EN ISO 13407. Although the definitions of base practices defined in the ISO/PAS 18152 are not considered as invalid they leave leeway for interpretation [9].

However, while this 'common understanding' seems true on a very abstract level, strong differences in how to implement these in practice can be expected. The key question therefore is not only what should be done, but rather how it can be assured that everything needed is being performed (or guaranteed) in order to gain a certain quality of a result, an activity or the process itself. In addition, the completeness and correctness of the base practices and human-centred design activities as defined in the ISO/PAS 18152 itself needed to be verified.

To establish such criteria based on current best practice the authors performed semi-structured interviews and questionnaires with six experts in the field of UE [10]. The experts were volunteers from an expert working-group on the integration of software engineering and usability engineering, the ISO committee TC 159 Ergonomics/SC4 as well as industry experts. A substantial part of the interviews referred explicitly to quality characteristics/aspects of the human-centered design activities of ISO 13407: 'context of use', 'user requirements', 'produce design solutions' and 'evaluation of use'. The goal was to identify what constitutes the quality of a certain activity from the experts' point of view and what kind of (potentially measurable) success and quality criteria exist that are relevant on a process level and subsequently for the implementation in practice.

As a result, overarching process- and quality characteristics were derived that led to statements about the relevance, the application and need of usability activities, methods and artifacts to be implemented in SE.

In summary, it can be said that the quality of the four activities essentially depends on the production and subsequent treatment of the result generated by each activity. 
From the quality perspective it is less important how something is accomplished, but rather to guarantee the quality of the results. In order to answer the question what constitutes this quality, the analyzed statements of the experts regarding each activity have been analyzed and documented. In [9] the core characteristic (essence) of each activity is described, followed by requirements regarding the generation and treatment of content, a summary of (measurable) quality criteria and success characteristics, as well as a list of operational measures that can be used for the implementation in practice.

After this, the authors categorized and structured each single statement (473 altogether) by: an unique id; its origin (question concerning one activity: 'context of use', 'user requirements', 'produce design solutions' or 'evaluation of use'); and the references to ISO 13407 and/or ISO/PAS 18152 (questions aimed on the goals and base practices of the two standards). After that, the statements have been categorized whether they addresses quality aspects regarding the process, an activity, or deliverable. All those parameters have been used to perform several iterations of analysis. By doing this each statement of each single interview partner has been compared with the statements of the others in order to count the number of similarities in terms of the content. Those statements that reflect a similar issue have been combined to a general requirement. As a result, the more statements refer to one requirement the more relevant it has been rated. By doing this, the statements have been fused and formalized into 107 'requirements for development processes or process models' that define the demands of UE (see Table 1 for an excerpt, complete list will be online for publication shortly). At last, the requirements have been categorized according to their focus on: quality aspects, the process, activities or deliverables.

As a result the authors distinguish two distinct types of requirements: Compliancy and key requirements. Compliancy requirements represent the goals and base practices defined in the standards and refine them with the input of the experts from the interviews. Key requirements define core characteristics of the overall frameworks usability activities focusing on the quality of activities and results.

The beneficial use of the requirements is two-sided: First, as a basis for the definition of software development processes aiming at usable solutions; and second, as an enhancement to the assessment standard ISO/PAS 18152 and its assessment criteria.

\section{Steps towards Validation and Application}

Validation of process changes in complex processes like software development is difficult because the cost and complexity of real world development projects makes controlled experiments impossible. To validate the compliancy and key requirements we used two approaches.

In the first step we distributed a questionnaire to 13 usability experts (again involving experts from industry, academia and ISO standardization committees) in which they were asked to rate the requirements from their practical experience with regards to correctness (is this requirement correct) and relevance (how important is the requirement with regards to the overall goal of ensuring usability). Table 1 shows these rating for exemplary requirements. As a result, compliancy and key requirements were established that represent an evaluated knowledge basis for the development of usable products $[8,10]$. 
Table 1. Examples of the requirements for the UE-activites 'context of use' (CoU), 'user requirements' (UR), 'produce design solutions' (PDS) and 'evaluation of use' (EoU) and the experts' rating in terms of correctness and relevance (in practice)

\begin{tabular}{|c|c|c|c|c|}
\hline $\mathrm{Nr}$ & Activity & Requirement & Correctness & Relevance \\
\hline 2 & $\mathrm{CoU}$ & $\begin{array}{l}\text { Context-analysis is an integral part of the } \\
\text { process. }\end{array}$ & Correct & Very high \\
\hline 17 & $\mathrm{CoU}$ & $\begin{array}{l}\text { The outcomes of the context analysis serve as } \\
\text { the input for the next process step and the } \\
\text { activity itself is anchored within the process } \\
\text { model accordingly. }\end{array}$ & Correct & High \\
\hline 27 & $\mathrm{CoU}$ & $\begin{array}{l}\text { The characteristics of the intended users and } \\
\text { their tasks, including user interaction with } \\
\text { other users } \\
\text { and other systems, are documented. }\end{array}$ & Correct & $\begin{array}{l}\text { Very } \\
\text { High }\end{array}$ \\
\hline 24 & $\mathrm{CoU}$ & $\begin{array}{l}\text { The analysis is focused on the original } \\
\text { context of the users (their goals, tasks, } \\
\text { characteristics of the tasks and the } \\
\text { environment, etc.). The analysis is } \\
\text { independent of any existing } \\
\text { solution/implementation. }\end{array}$ & Correct & High \\
\hline 33 & $\mathrm{CoU}$ & $\begin{array}{l}\text { The context-information is based on facts and } \\
\text { not an interpretation of any situation. }\end{array}$ & Sufficient & Medium \\
\hline 46 & UR & $\begin{array}{l}\text { A sufficient amount of user requirements are } \\
\text { the basis for the next process step (PDS). }\end{array}$ & Correct & $\begin{array}{l}\text { Very } \\
\text { High }\end{array}$ \\
\hline 71 & PDS & $\begin{array}{l}\text { The development of solutions is carried out in } \\
\text { collaboration with the development team. }\end{array}$ & Correct & $\begin{array}{l}\text { Very } \\
\text { High }\end{array}$ \\
\hline
\end{tabular}

In a second step we examined in how far the requirements are suitable to identify problems with existing processes as documented in literature. While a notable scarcity of such documented experiences exists there are some useful exceptions. In particular Jokela [5] has conducted and documented eleven process assessments within the last years and identified a number of common usability problems in industrial practice, e.g. Missing or Only Partial Usability Engineering Activities, Results of Usability Activities Not Considered, Quality Problems, Knowledge and Skills Problems, etc. By matching these problems identified by Jokela in 11 assessment projects with the compliancy and key requirements, we established that the majority of the reported problems are addressed by the key requirements. This indicates that common problems of UE in development practice could be avoided if the compliance and key requirements were considered during the definition of development processes.

The requirements could also be used as detailed criteria for assessing processes (or process models) leading to more specific results about weaknesses. In addition, more specific measures for improvement could be derived than in common assessment approaches (i.e. the ISO/PAS 18152).

The compliancy and key requirements therewith not only reflect basic demands to be applied on development processes they also address common problems of integrating SE and UE. By fulfilling these requirements a substantial contribution to the development of usable solutions could be made. 
Altogether the requirements lead to the integration of SE and UE on all three levels of abstraction. They define goals and characteristics of activities, quality and success criteria as well as results that lead to the systematic development of usable products. This is reflected through the overarching framework and therewith on the level of standards, by the requirements' ability to be applied on the level of process models and the operational level in which the models are tailored to fit the specifics of an organization.

\section{Conclusion and Outlook}

This paper identifies compliancy and key requirements for the integration of usability engineering activities into software engineering processes. The 107 requirements define objectives and characteristics of activities as well as quality and success criteria for execution and were ranked and rated by usability engineering experts to provide an indication of their validity and relevance. The requirements are abstract enough to serve as assessment criteria for a wide range of processes but their application in an assessment process provides more specific guidance than generic guidelines. The requirements can be used to refine to the ISO/PAS 18152, a public available specification for a process assessment of human-system issues.

Currently the authors are analyzing the changes in the new version of the standard ISO 9241-210 (which replaced the ISO 13407) and are going to adapt the requirements where necessary. In addition the ISO/IEC 12207 is being operationalized in more detail (activities, tasks, artifacts and their relations) and are mapped to the compliancy and key requirements. The results will end in a new reference model for integrating software engineering and usability engineering likewise. The model can be used for process definition and assessments as well. A first expert based evaluation of the model is going to be finished very soon.

In future work the authors aim to apply the optimized assessment approach to additional process models, such as agile approaches, to establish their suitability beyond traditional SE processes.

\section{References}

1. DIN EN ISO 13407: Human-centered design processes for interactive systems, CEN European Committee for Standardization, Brussels (1999)

2. ISO/IEC 12207: Information technology - Software life cycle processes, 2nd ed. 2008-0201. ISO/IEC, Genf (2008)

3. ISO/IEC 15504: Information technology - Process Assessment - Part 1-5. ISO/IEC, Genf (2003-2006)

4. ISO/PAS 18152: Ergonomics of human-system interaction - Specification for the process assessment of human-system issues. ISO, Genf (2003)

5. Jokela, T.: Performance Rather than Capability Problems. Insights from Assessments of Usability Engineering Processes. In: Bomarius, F., Komi-Sirviö, S. (eds.) PROFES 2005. LNCS, vol. 3547, pp. 115-127. Springer, Heidelberg (2005) 
6. Nebe, K., Zimmermann, D.: Suitability of Software Engineering Models for the Production of Usable Software. In: Gulliksen, J., Harning, M.B., van der Veer, G.C., Wesson, J. (eds.) EIS 2007. LNCS, vol. 4940, pp. 123-139. Springer, Heidelberg (2008a)

7. Nebe, K., Zimmermann, D.: Aspects of Integrating User Centered Design to Software Engineering Processes. In: Jacko, J.A. (ed.) HCI 2007. LNCS, vol. 4550, pp. 194-203. Springer, Heidelberg (2007)

8. Nebe, K., Paelke, V.: Usability-Engineering-Requirements as a Basis for the Integration with Software Engineering. In: Jacko, J.A. (ed.) HCII 2009, Part I. LNCS, vol. 5610, pp. 652-659. Springer, Berlin (2009)

9. Nebe, K., Zimmermann, D., Paelke, V.: Integrating Software Engineering and Usability Engineering. In: Pinder, S. (ed.) Advances in Human-Computer Interaction, ch. 20, pp. 331-350. I-Tech Education and Publishing, Wien (2008)

10. Nebe, K.: Integration von Usability Engineering und Software Engineering: Konformitäts- und Rahmenanforderungen zur Bewertung und Definition von Softwareentwicklungsprozessen, Doctoral Thesis, Shaker Verlag, Aachen (2009)

11. Pawar, S.A.: A Common Software Development Framework For Coordinating Usability Engineering and Software Engineering Activities. Master Thesis, Blacksburg, Virginia (2004)

12. Pelka, A.I.: Integration von SW- und Usability-Engineering am Beispiel des V-Modells. Diplomarbeit, Rheinische Friedrich-Wilhelms-Universität, Bonn (2001)

13. Rechenberger, P., Pomberger, G.: Informatik-Handbuch. Carl Hanser Verlag, München (2002)

14. Schaffer, E.: Institutionalization of usability: a step-by-step guide. Addison-Wesley; Pearson Education, Inc., Boston (2004)

15. Seffah, A., Desmarias, M.C., Metzker, E.: Human Centered Software Engineering, HCI, Usability and Software Engineering Integration: Present and Future. In: Seffah, A., Gulliksen, J., Desmarais, M.C. (eds.) Human-Centred Software Engineering - Integrating Usability in the Development Lifecycle, vol. 8. Springer, Berlin (2005) 